Europhys. Lett., 69 (2), pp. 163-169 (2005)

DOI: $10.1209 / \mathrm{epl} / \mathrm{i} 2004-10332-1$

\title{
Classical robustness of quantum unravellings
}

\author{
D. J. Atkins ${ }^{1,2}$, Z. Brady ${ }^{1}$, K. JACOBS ${ }^{1,2}$ and H. M. Wiseman ${ }^{1,2}$ \\ 1 Centre for Quantum Dynamics, School of Science, Griffith University \\ Brisbane, 4111 Queensland, Australia \\ 2 Centre for Quantum Computer Technology - Australia
}

received 9 August 2004; accepted in final form 10 November 2004

published online 22 December 2004

PACS. 03.65.Yz - Decoherence; open systems; quantum statistical methods.

PACS. 42.50.Lc - Quantum fluctuations, quantum noise, and quantum jumps.

PACS. 05.40.Jc - Brownian motion.

\begin{abstract}
We introduce three measures which quantify the degree to which quantum systems possess the robustness exhibited by classical systems when subjected to continuous observation. Using these we show that for a fixed environmental interaction the level of robustness depends on the measurement strategy, or unravelling, and that no single strategy is maximally robust in all ways.
\end{abstract}

When describing the observation of individual quantum systems, it is crucial to explicitly treat the measurement process using quantum measurement theory. The reverse, however, is commonly true for classical systems - it is quite usual for experimentalists both to model and gather data about classical systems without any reference to classical measurement theory (being the theory of Bayesian statistical inference or nonlinear filtering [1,2]). This dramatic contrast is only possible because of certain properties which many classical systems possess. Three such properties are apparent if we consider watching the motion of a pendulum: 1) when we open our eyes, we obtain the information (the location and velocity of the pendulum) almost instantaneously; 2) the information we obtain is not exclusive - many people can observe the same system, and will all agree upon the results; and 3) the system is relatively unaffected by noise so that if we close our eyes for a moment, we can accurately predict what we will see when we open them again $\left({ }^{1}\right)$. We will refer to the degree to which a system possesses these properties as its degree of robustness under each property.

The classical robustness of quantum systems in this sense was the subject of a recent study by Dalvit, Dziamarga and Zurek [3]. They concluded that, for a given environmental interaction, a single measurement strategy will maximize the robustness for both properties 1) and 3) above. Here we investigate the degree of robustness of two canonical quantum systems for the three properties above (and a fourth), under a wide class of measurement strategies. We show that this conclusion is, in fact, not warranted. That is, in general, for a fixed environmental interaction, different measurement strategies are required to maximize

$\left({ }^{1}\right)$ Note that the existence of chaos in a system does not destroy its robustness: in this case, the divergence of neighboring trajectories is exponential and thus has a negligible effect on the predictability for short times.

(c) EDP Sciences 
different notions of classical behavior, and that this depends not only on the system in question, but on the dynamical regime. There is thus necessarily a trade-off between different types of robustness. While this study is of fundamental interest, it is also of practical importance for the feedback control of quantum systems [4-6], as will be discussed.

To clarify the above and establish our results, it is useful first to establish some concepts and terminology. The continuous observation of a quantum system can always be described by treating the interaction of the system in question with an environment consisting of a large number of degrees of freedom. As a result of the interaction, the environment continuously extracts information about the system, and this information can be read by measuring the state of the environment. This has been called quantum filtering [7]. Once one has chosen an interaction, one therefore has the remaining freedom to choose the manner in which to interrogate the environment, and different methods lead to qualitatively different kinds of measurements. These different measurements, which constitute the measurement strategies discussed above, are often referred to as different unravellings of the environmental interaction [8].

In what follows, by conditional evolution, or trajectory, we will mean the evolution of the system as a result of a particular unravelling $(\mathcal{U})$ and by unconditional evolution we will mean the evolution of the system under the same environmental interaction, but without the measurement of the environment. The unconditional evolution is simply given by the ensemble average $(E[\cdot])$ over all the possible conditioned evolutions.

Before introducing quantitative measures of robustness which characterize the speed, nonexclusivity and predictability of a system, we briefly revisit the conclusion of ref. [3]. Using our terminology, they maintained that the unravelling that most rapidly purifies the state is identical to the one that will tend to collapse the system into a "pointer state" $\left({ }^{2}\right)$. The "pointer states" [10] are those that lose their purity most slowly when the environment is unobserved. If true, this would imply that the same unravelling is most robust under our properties 1) and 3). Reference [3] also argued that purity loss and fidelity loss were equivalent for the purposes of defining robustness. We show that in general, contrary to both of the above conclusions, different unravellings are optimal for these various concepts of robustness.

Measures of robustness of unravellings. - We will consider four quantitative measures of robustness. The first, purification time, quantifies the rate at which the measurement provides information about the system. To define this we allow the system to evolve to its unconditional steady state, and then ask how long it takes, upon switching on the observation, for the measurement to increase the purity halfway to its long-time value (which, for an efficient measurement, is unit purity). Formally, the purification time, $\tau_{\text {pur }}$, is the earliest solution of

$$
E\left[\operatorname{Tr}\left[\left\{\mathcal{S}_{1}^{\mathcal{U}}(\tau, 0) \rho_{\mathrm{ss}}\right\}^{2}\right]\right]=\theta,
$$

where $\theta \equiv \frac{1}{2}\left(1+\operatorname{Tr}\left[\rho_{\mathrm{ss}}^{2}\right]\right)$. Here $\rho_{\mathrm{ss}}$ is the unconditional steady state of the system and $\mathcal{S}_{\eta}^{\mathcal{U}}\left(t_{2}, t_{1}\right)$ is the nonlinear, stochastic, completely positive map which takes the system from time $t_{1}$ to $t_{2}$ during a measurement of efficiency $\eta$. It has the properties

$$
E\left[\mathcal{S}_{\eta}^{\mathcal{U}}(t, 0)\right] \equiv \mathcal{O}(t, 0)=\mathcal{S}_{0}^{\mathcal{U}}(t, 0)=e^{\mathcal{L} t},
$$

where $\mathcal{L}$ is the Lindblad-type superoperator generating the unconditional evolution.

The purification time is relevant for the feedback control of quantum systems. Feedback control is realized by observing a system and using this information, as it is obtained, to control the evolution (usually by modifying the system Hamiltonian). The effectiveness of

\footnotetext{
$\left({ }^{2}\right)$ We note that a "pointer state" unravelling similar to this was previously considered in ref. [9].
} 
any feedback algorithm is thus ultimately limited by the extent to which the system state is known. The purification time therefore provides an indication of the time required for a feedback loop to become effective, when it employs the given measurement strategy.

Secondly, we wish to quantify the extent to which the existence of multiple observers interferes with the ability of each observer to track the system. In order for multiple parties to observe the system separately, they must divide up the environment between them. This looks, to one of the observers, $i$, as if she has an inefficient measurement, with efficiency coefficient $\eta_{i}$, such that $\sum_{i} \eta_{i}=1[11,12]$. We will quantify the robustness of the measurement scheme against a division of the environment by asking at what value of efficiency the average purity of the long-time observed state is halfway between its value at perfect efficiency, and that of no observation at all. We will refer to this as the efficiency threshold, and denote it by $\eta_{\text {thr }}$; formally, this is the value of $\eta$ such that

$$
\lim _{t \rightarrow \infty} E\left[\operatorname{Tr}\left[\left\{\mathcal{S}_{\eta}^{\mathcal{U}}(t, 0) \rho_{\mathrm{ss}}\right\}^{2}\right]\right]=\theta
$$

The smaller $\eta_{\text {thr }}$ is, the more robust a feedback algorithm would be in the face of inefficiency.

Our third measure of classical robustness, mixing time, quantifies the rate at which the system becomes unpredictable - essentially it characterizes the sensitivity of the system to noise from its environment. To define the mixing time, we allow the observed trajectory to evolve for long enough that the state is pure, and that the unconditioned evolution would have reached a steady state, stop measuring the environment, and consider the decrease in the purity of the state as time passes. The mixing time is the time at which the purity falls halfway from its initial value (being unity) to the value it would take if the system were allowed to evolve back to its steady state (being $\operatorname{Tr}\left[\rho_{\mathrm{Ss}}^{2}\right]$ ). Formally, the mixing time, $\tau_{\text {mix }}$, is the earliest solution of

$$
\lim _{t \rightarrow \infty} E\left[\operatorname{Tr}\left[\left\{\mathcal{O}(t+\tau, t) \mathcal{S}_{1}^{\mathcal{U}}(t, 0) \rho_{\mathrm{ss}}\right\}^{2}\right]\right]=\theta .
$$

This quantifies the predictability of the evolution while the observer is not looking.

A related concept, introduced previously by two of us and Vaccaro $[13,14]$, is the extent to which an initially conditioned state remains unchanged during a period of unconditional evolution. This is quantified by the survival time, $\tau_{\text {sur }}$, the earliest solution of

$$
\lim _{t \rightarrow \infty} E\left[\operatorname{Tr}\left[\mathcal{S}_{1}^{\mathcal{U}}(t, 0) \rho_{\mathrm{SS}} \times \mathcal{O}(t+\tau, t) \mathcal{S}_{1}^{\mathcal{U}}(t, 0) \rho_{\mathrm{ss}}\right]\right]=\theta
$$

Note that the stochastic map $\mathcal{S}_{1}^{\mathcal{U}}(t, 0)$ appearing twice in this equation is the same map (i.e. has the same noise). We also note that $\tau_{\text {sur }} \leq \tau_{\text {mix }}$. In general, this does not measure the noise-sensitivity alone, since any deterministic evolution will contribute to changing the state. While the survival time is not motivated by classicality like the other measures of robustness, it is, like them, relevant for feedback control. Consider feedback with a time delay $\tau$. For a feedback algorithm designed for $\tau=0$, the performance should not be greatly degraded as long as $\tau \lesssim \tau_{\text {sur }}$, as the system will not have strayed significantly during the delay. On the other hand, deterministic evolution during the delay could be corrected for in the algorithm design. In that case, the performance of the feedback control should not be significantly worse than for the case of no delay as long as $\tau \lesssim \tau_{\text {mix }}$.

A particle undergoing quantum Brownian motion. - We consider a particle in one dimension with position $q$ and momentum $p$ in a viscous environment at temperature $T$. For our first example we will consider all continuous Markovian unravellings [15]. In an optical 
realization, this includes all homodyne and heterodyne detection schemes [15]. The stochastic master equation (SME) describing the conditional evolution of a particle under quantum Brownian motion (QBM), for all such unravellings, is

$$
\begin{aligned}
\mathrm{d} \rho_{\mathrm{c}}= & -(i / 2)\left[p^{2}+(q p+p q) / 2, \rho_{\mathrm{c}}\right] \mathrm{d} t+\mathcal{D}[c] \rho_{\mathrm{c}} \mathrm{d} t+ \\
& +\left\{\sqrt{\eta} \mathrm{d} W\left(c-\operatorname{Tr}\left[c \rho_{\mathrm{c}}\right]\right) \rho_{\mathrm{c}}+\text { H.c. }\right\}
\end{aligned}
$$

Here $c \equiv \sqrt{2 T} q+i p / \sqrt{8 T}$ and

$$
\mathcal{D}[B] A=B A B^{\dagger}-\frac{1}{2}\left(B^{\dagger} B A-A B^{\dagger} B\right)
$$

The subscript $\mathrm{c}$ indicates that the evolution is conditioned on the innovation [1] $\mathrm{d} W$, a stochastic Wiener increment (to which we will return below). Finally, note that we are using scaled units such that the damping rate $\gamma$, the particle mass $m$, and $\hbar$ are all unity. Averaging over the noise (or setting the detection efficiency $\eta$ to zero) removes the second line in eq. (6), leaving a version of the QBM master equation which is in the Lindblad form [16]. It is the standard time-independent QBM master equation [17], with the necessary addition of a term which generates position diffusion [18]. This system is also equivalent to the damping of an optical cavity mode with an added nonlinearity.

The Wiener increment in the above SME satisfies $\mathrm{d} W \mathrm{~d} W^{*}=\mathrm{d} t$ and $\mathrm{d} W^{2}=v \mathrm{~d} t$, where $v=r e^{i \phi} \in \mathbb{C}$ with $r \leq 1$. It is the value of $v$ which determines the measurement strategy, and thus our search for optimally robust unravellings will involve optimization over the disk parametrized by $r$ and $\phi$. In an optical realization, $r=0$ corresponds to heterodyne detection, and $r=1$ corresponds to homodyne detection of a linear combination of $q$ and $p, x=$ $c e^{-i \phi / 2}+c^{\dagger} e^{i \phi / 2}(\phi=0$ corresponds to a measurement of $q$ and $\phi=\pi$ to one of $p)$.

Since the unconditional $(\eta=0)$ steady state of eq. (6) possesses a Gaussian Wigner function, and the conditional evolution preserves Gaussianity, the four measures of robustness with which we are concerned may be written solely in terms of the variances of $q$ and $p$ and their covariance $C_{q p} \equiv\langle q p+p q\rangle / 2-\langle q\rangle\langle p\rangle$. In particular, the purity, which is required for three of the four measures, is $P=1 / \sqrt{4\left(V_{q} V_{p}-C_{q p}^{2}\right)}$. Furthermore, these quantities evolve deterministically even under conditional stochastic evolution. This is very useful, as it removes the need to do stochastic simulations for the ensemble averages in eqs. (1)-(5). This enables us to numerically optimize robustness over all the measurement strategies (all values of $v$ ).

Optimizing for each form of robustness over the disc of measurement strategies, we find that the optimal strategy always lies on the boundary of the disc $(r=1)$, and thus corresponds to some form of homodyne detection. However, the type of homodyne detection required (that is, the value of the phase $\phi$ ) is highly dependent upon the type of robustness desired. The results are summarized in fig. 1, where the optimal phase for each notion of robustness is plotted as a function of the temperature of the bath. We see that while the purification time and efficiency threshold may be optimized more or less simultaneously, the mixing and survival times require quite different unravellings $\left({ }^{3}\right)$.

$\left({ }^{3}\right)$ It is worth noting a subtlety regarding the optimal strategy for the survival time at large temperatures. While it is true that setting $\phi=\pi$ results in a homodyne measurement of momentum, it is incorrect to conclude from fig. 1 that the optimal survival time is obtained by a momentum measurement for large $T$. This is because, for values of $\phi$ close to $\pi$, the measured observable is given by $x \sim-\frac{1}{\sqrt{8 T}} p+(\pi-\phi) \frac{\sqrt{2 T}}{2} q$. For large $T$, the $\phi$ which maximizes $\tau_{\text {sur }}$ is such that $(\pi-\phi) \sim T^{-1 / 3}$. Thus $x$ is, in fact, dominated by position, as for the other measures. 


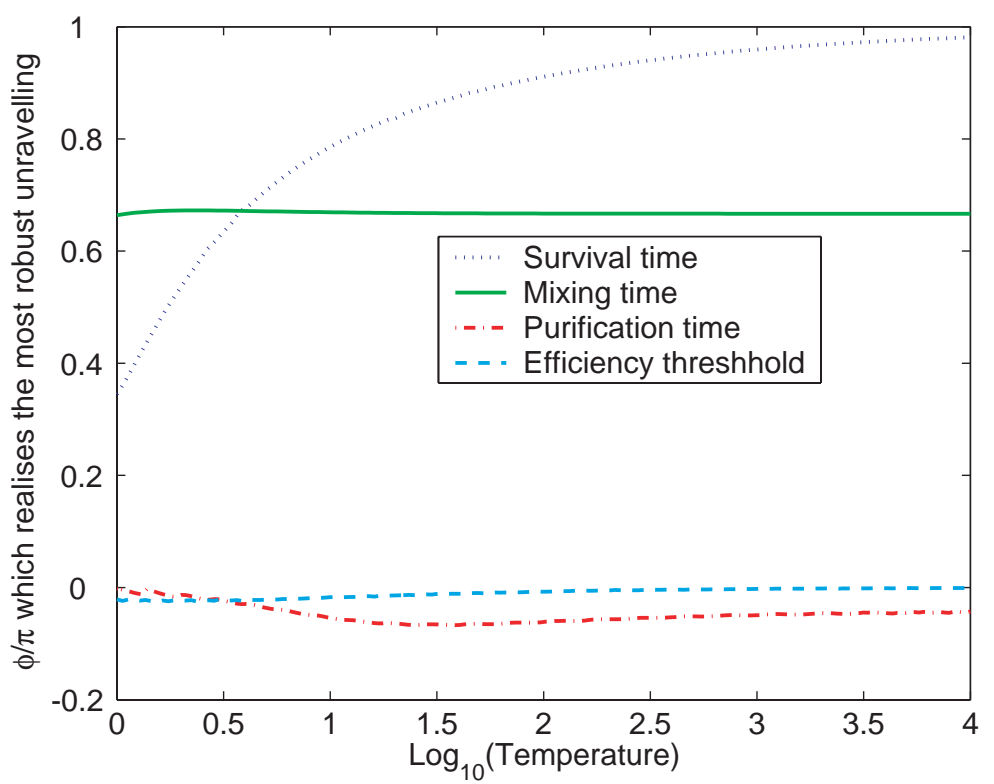

Fig. 1 - The angle, $\phi$, which gives the optimally robust measurement scheme for each of the four forms of robustness, plotted as a function of the dimensionless temperature.

The two-level atom. - The unconditional evolution of our second example, a driven two-level atom (TLA), is governed by the resonance fluorescence master equation

$$
\frac{\mathrm{d} \rho}{\mathrm{d} t}=-i \frac{\Omega}{2}\left[\sigma_{x}, \rho\right]+\mathcal{D}\left[\sqrt{\gamma} \sigma_{-}\right] \rho,
$$

where $\Omega$ is the Rabi frequency and $\gamma$ is the spontaneous-emission rate. Since the electromagnetic field is the bath which mediates the measurement, all measurement strategies involve observing the atomic radiation. The equation describing a particular unravelling will include additional stochastic terms specific to the unravelling. Note that unconditional dynamics of the TLA are entirely determined by the dimensionless parameter $\Omega / \gamma$.

For the TLA we will consider both continuous unravellings and those which involve discontinuous jumps. However, in this case we cannot eliminate the need to take ensemble averages over large numbers of trajectories (for the results which follow, typically hundreds of thousands). As we are not therefore able to optimize over all unravellings, we choose a small set which are most relevant, either from an experimental point of view, or because they possess special properties. We consider direct photon counting, homodyne $x$ detection $(\phi=0)$, homodyne $y$ detection $(\phi=\pi)$, heterodyne detection, and Adaptive Interferometric Detection (AID). This last measurement scheme was introduced by Wiseman and Toombes [19], and involves interference of the emitted radiation with a local oscillator (LO) as in homodyne detection. However, unlike in homodyne detection, the LO is weak (comparable in amplitude to the TLA field) so that individual photons are resolvable, resulting in a jump process. Upon each jump the amplitude of the LO is flipped via a real-time feedback loop, which makes it adaptive and non-Markovian. We consider AID because it has been shown in ref. [14] that it is the optimal unravelling for maximizing the survival time, and is thus a likely candidate for maximizing the other measures of robustness in which we are interested here. 
TABLE I - Rankings of robustness of unravellings for each measure of robustness with most robust at the top down to least robust at the bottom.

\begin{tabular}{llllll}
\hline$\tau_{\text {sur }}^{\mathcal{U}}$ & $\tau_{\text {mix }}^{\mathcal{U}}$ & $\begin{array}{l}\tau_{\text {pur }}^{\mathcal{U}} \\
\Omega \lesssim \gamma / 2\end{array}$ & $\begin{array}{l}\tau_{\text {pur }}^{\mathcal{U}} \\
\Omega \gtrsim 4 \gamma\end{array}$ & $\begin{array}{l}\eta_{\text {thr }}^{\mathcal{U}} \\
\Omega \simeq \gamma / 2\end{array}$ & $\begin{array}{l}\eta_{\text {thr }}^{\mathcal{U}} \\
\text { ¿ }\end{array} 4 \gamma$ \\
\hline AID & AID & Hom. $x$ & Het. & AID & AID \\
Hom. $x$ & Hom. $x$ & AID & Hom. $y$ & Hom. $x$ & Het. \\
Het. & Het. & Direct & Hom. $x$ & Direct & Hom. $x$ \\
Hom. $y$ & Hom. $y$ & Het. & AID & Het. & Hom. $y$ \\
Direct & Direct & Hom. $y$ & Direct & Hom. $y$ & Direct \\
\hline
\end{tabular}

We now evaluate the three measures of classical robustness for each of the above measurement schemes, to rank them. The fourth robustness measure, the survival time, has been calculated for this system previously in [14], and we include these results here for comparison. The rankings of the unravellings are displayed in table I, from most to least robust.

The rankings of the unravellings determined by the survival and mixing times are identical, and independent of $\Omega / \gamma$; AID remains the most robust by these measures for all dynamical regimes. The ranking under purification time depends on the regime. For weak driving $(\Omega \lesssim \gamma / 2)$, homodyne- $x$ and AID provide the most rapid means of obtaining information about the system. However, for strong driving AID is one of the least effective at extracting information. The full dependence of the purification time for all the schemes is displayed in fig. 2. The ranking in terms of the efficiency threshold also depends on the dynamical regime, as can be seen in table I. For $\gamma \ll \Omega$ (not shown in the table) direct detection actually gives the most robust efficiency threshold.

Discussion. - We have introduced means of quantifying the degree to which quantum systems behave in a classical fashion under continuous observation. We have also investigated, for two canonical systems, how this classicality or robustness depends on the way in which the environment is interrogated.

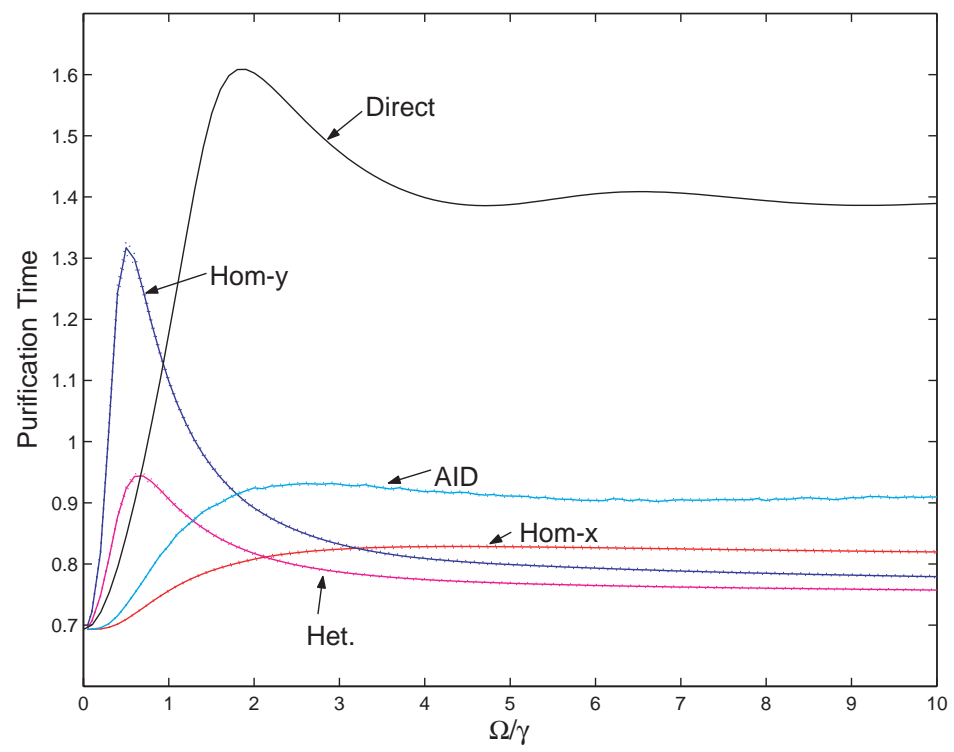

Fig. 2 - The purification time (in units of $\gamma^{-1}$ ) for different measurement schemes. 
Reviewing the results for both systems reveals that for the most part, of all the Markovian measurement schemes, homodyne detection provides the most classically robust means of observing the systems. However, for QBM the different concepts of robustness require different homodyne schemes (i.e. different $\phi$ ). For the TLA, an adaptive (hence non-Markovian) measurement strategy is most robust in general. Moreover, for the TLA, there are dynamical regimes (when looking at purification time and efficiency threshold) where direct and heterodyne detection render the most robust behavior. In summary, it is clear that there is no unique unravelling which is the most classically robust, contrary to previous expectations [3].

The notions of robustness which we have considered here also provide an indication of the relative merits of different measurement schemes for feedback control constrained by time delays and measurement inefficiency. The fact that no single unravelling is maximally robust in all ways suggests that the measurement strategy adopted for the purposes of feedback control will need to be tailored to the robustness requirements of a given application. Quantifying this link between classical robustness and quantum control should provide useful insight into the design of quantum feedback algorithms.

$$
* * *
$$

This work was supported by the Australian Research Council and the State of Queensland. HMW thanks G. Toombes for formative discussions many years ago.

\section{REFERENCES}

[1] Box G. E. P. and Tino G. C., Bayesian Inference in Statistical Analysis (Addison-Wesley, Sydney) 1973.

[2] Maybeck P. S., Stochastic Models, Estimation, and Control, Vol. III (Academic Press, New York) 1982.

[3] Dalvit D. A. R., Dziarmaga J. and Zurek W. H., Phys. Rev. Lett., 86 (2001) 373.

[4] Belavkin V. P., Rep. Math. Phys., 43 (1999) 405.

[5] Doherty A. C. and Jacobs K., Phys. Rev. A, 60 (1999) 2700; Doherty A. C., Habib H., Jacobs K., Mabuchi H. and Tan S. M., Phys. Rev. A, 62 (2000) 012105.

[6] Wiseman H. M., Mancini S. and Wang J., Phys. Rev. A, 66 (2000) 013807.

[7] Belavkin V. P., Lecture Notes in Control and Information Sciences, edited by BlaquiÈre A., Vol. 121 (Springer, Berlin) 1988, p. 245.

[8] Carmichael H. J., An Open Quantum Systems Approach to Quantum Optics (Springer, Berlin) 1993.

[9] Diósi L. and Kiefer C., Phys. Rev. Lett., 85 (2000) 3552.

[10] Zurek W. H., Habib S. and Paz J. P., Phys. Rev. Lett., 70 (1993) 1187.

[11] Barchielli A., Int. J. Theor. Phys., 32 (1993) 2221.

[12] Dziarmaga J., Dalvit D. A. R. and Zurek W. H., Phys. Rev. A, 69 (2004) 022109.

[13] Wiseman H. M. and Vacarro J. A., Phys. Lett. A, 250 (1998) 241; Phys. Rev. A, 65 (2002) 043606.

[14] Wiseman H. M. and Brady Z., Phys. Rev. A, 62 (2000) 023805.

[15] Wiseman H. M. and Diosi L., Chem. Phys., 268 (2001) 91.

[16] Lindblad G., Commun. Math. Phys., 48 (1976) 199.

[17] Gardiner C. W. and Zoller P., Quantum Noise (Springer-Verlag, Berlin) 2000.

[18] Diosi L., Europhys. Lett., 22 (1993) 1.

[19] Wiseman H. M. and Toombes G. E., Phys. Rev. A, 60 (1999) 2474. 